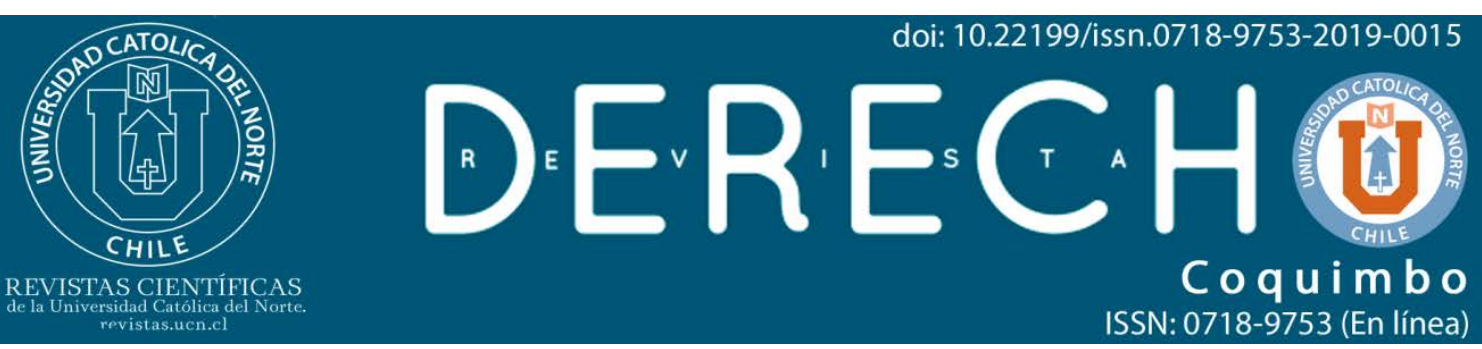

\title{
Déficits en la medición del miedo al delito en Chile
}

\section{Deficits in the measurement on crime fear in Chile}

Sebastián Galleguillos Agurto* (D) https://orcid.org/0000-0001-5772-637X

*Universidad de Talca, Chile. Investigador. CEDEP. Abogado. Lic. en Cs. Jurídicas y Sociales, U. de Chile. sgalleguillos@utalca.cl

(cc) BY

\section{Resumen:}

El propósito es detectar una serie de dificultades conceptuales y metodológicas en la investigación chilena sobre miedo al delito. Para llevar a cabo lo anterior, se realiza a modo de introducción un apartado sobre las principales dimensiones del miedo al delito, para con posterioridad describir las problemáticas que la literatura comparada ha detectado sobre la forma tradicional de medir dicho fenómeno. El documento finaliza delineando sugerencias para futuras investigaciones en el contexto chileno

Palabras Clave: Miedo al delito; Metodología cualitativa; Metodología cuantitativa; Populismo punitivo.

\begin{abstract}
:
The purpose is to identify a series of conceptual and methodological difficulties in Chilean research on crime fear. In order to carry out the aforementioned, the introduction includes a section regarding the main dimensions of crime fear, in order to describe the problems that comparative literature has detected on the traditional way of measuring said phenomenon. The document ends by outlining suggestions for future research in the Chilean context.

Keywords: Fear of crime; Quantitative research; Qualitative research; Punitive populism.
\end{abstract}


"There's always fear of the unknown where there's mystery. It's possible to achieve a state where you realize the truth of life and fear disappears, and a lot of people have reached that state, but next to none of them are on Earth"

\section{Problemas de raíz: múltiples dimensiones y efectos del miedo al delito}

Hoy en día el miedo al delito sigue constituyendo un tópico con alcances difusos, que no posee un concepto delimitado y que adolece de profundos problemas metodológicos al momento de ser investigado (Gray, Jackson y Farrall, 2011, pp. 3 y 4). Pese a dichas dificultades, el interés que reviste esta temática es amplia, atrayendo indistintamente la atención de políticos y académicos (Gray, Jackson y Farrall, 2011, p. 2), pudiendo encontrarse perspectivas de análisis provenientes tanto del mundo sociológico como desde los estudios criminológicos (Cornelli, 2008, p. 7), complementadas incluso recientemente con miradas desde la micro-economía (Canare, Francisco y Jopson, 2017, pp. 4 y ss.).

Considerando esta variedad de perspectivas y focos de análisis, el principal objetivo del presente documento es abocarse a la detección de una serie de déficits metodológicos y conceptuales en la medición del miedo al delito circunscrita al ámbito chileno. Para contextualizar dicho diagnóstico, en este apartado introductorio se llevará a cabo una breve síntesis sobre los orígenes de su medición, algunas distinciones conceptuales relevantes para efectos de su discusión en Chile, y las consecuencias que reviste este fenómeno, teniendo en especial consideración las consecuencias político-criminales que reviste.

Sobre los orígenes de la investigación al miedo al delito, no resulta trivial que las primeras incursiones sobre miedo al delito llevadas a cabo en la década de 1960 no hayan tenido como finalidad la medición con bases conceptuales predefinidas sobre dicho fenómeno, sino que tenían por objetivo más bien recabar cifras negras sobre el delito en Estados Unidos (Narváez Mora, 2015, p. 96). Estas investigaciones iniciales dieron cuenta de la discordancia entre los niveles de victimización y riesgo percibido, marcando el escenario futuro sobre la racionalidad de las ansiedades que circundan el miedo al delito (Gray, Jackson y Farrall, 2011, p. 3). Con posterioridad a dichos estudios se ha desarrollado un debate que sigue vigente en la actualidad, tanto a nivel metodológico, terminológico y conceptual, llegando incluso a afirmarse la existencia de un "caos" en estos últimos dos niveles de análisis (Cornelli, 2008, p. 102).

A nivel terminológico, la principal distinción se encuentra establecida entre la expresión miedo al delito ("fear of crime") e inseguridad ciudadana. La inseguridad ciudadana sería un concepto genérico que abarcaría las preocupaciones inmersas en el 
discurso construido en torno a la sociedad del riesgo, incluyendo además de la delincuencia problemas que van desde la llegada de inmigrantes hasta el mismo cambio climático (Vozmediano Sanz, San Juan Guillén y Vergara Iraeta, 2008, p. 2).

Por otro lado, al concepto de miedo al delito se le han atribuido una heterogeneidad de significados, lo que en parte puede ser explicado por corresponder a un término usado en el lenguaje cotidiano presente en conversaciones casuales (Skogan, 1999, p. 47). Una de las primeras definiciones acuñadas en la literatura corresponde a la apuntada por Garofalo (1981, p. 840), quien define el miedo al delito como una reacción emocional, caracterizada por implicar una sensación de miedo de peligro y ansiedad. Es precisamente esta característica como "experiencia subjetiva negativa de carácter emocional", la que ha estado presente transversalmente en las elaboraciones de los autores (Narváez Mora, 2015, p. 99 y 103). Este rasgo como experiencia individual y subjetiva resultará fundamental en el análisis de las metodologías empleadas para la medición del miedo al delito.

Dentro del universo sobre percepciones del crimen, en un comienzo la literatura especializada distinguió entre un aspecto cognitivo y otro afectivo del miedo al delito. Estos, a su vez, pueden tener como referencia un marco general (orientado hacia la comunidad) o personal, pudiendo experimentarse reacciones que incluyen juicios, valores y emociones (Ferraro y Lagrange, 1987, pp. 71-72). Los juicios corresponden a una evaluación sobre el riesgo, los valores a "preocupación" sobre el delito, y las emociones "miedo" al delito. En base a este marco teórico, hoy en día se vincula el aspecto cognitivo a la evaluación de la probabilidad del riesgo, mientras que la dimensión afectiva se relaciona con la preocupación que genera el delito.

Prontamente se amplió este espectro de elementos, añadiéndose también la conducta desplegada por los individuos. Dentro de las conductas, se engloban tanto las acciones que las personas llevan individualmente para evitar situaciones peligrosas, como también las tácticas desarrolladas para mejorar la protección de sus hogares (Skogan, 1999, p. 48).

Con todo, no solo el concepto de fear of crime ha sido explorado por estudios comparados. La noción de anger of crime también ha sido incursionada en menor medida dentro de la experiencia comparada ${ }^{1}$, buscando diferenciar y precisar cuáles son las reacciones precisas en torno al fenómeno delictual. Si bien resulta difícil entregar una definición de anger of crime, de acuerdo a las investigaciones empíricas este concepto se encontraría vinculado a sentimientos de injusticia, abarcando una amplia variedad de emociones que recorren la irritación, molestia u odio (Farrall, 2004, pp. 162-163). A tal punto ha llegado la relevancia del anger of crime dentro de

\footnotetext{
${ }^{1}$ Sobre el cuestionamiento de porqué ha sido opacada la investigación de anger of crime por fear of crime, se han trazado diversas posibles explicaciones. Al respecto, véase Ditton, Farrall, Bannister, Gilchrist y Pease (1999, p. 51).
}

Rev. derecho (Coquimbo, En línea) 2019, 26: e3860 
dichos estudios, que incluso se ha concluido que los niveles de enojo serían mayores que los de temor (Ditton, Bannister, Gilchrist y Farrall, 1999, p. 98)².

En esta línea, mediante la inclusión de métodos cualitativos ${ }^{3}$ como entrevistas semi-estructuradas, se ha permitido que lo sujetos de estudio diferencien y precisen que sienten enojo en vez de miedo ante ciertas prácticas cotidianas (Farrall, Jackson y Gray, 2009, p. 71) ${ }^{4}$. Es importante destacar que los estudios sobre esta variedad de reacciones no suponen un punto de partida conceptual excluyente en cuanto a la exploración de los demás términos (Klama y Egan, 2011) 5 .

Los diversos niveles de complejidad y superposición recientemente descritos suelen encontrar un aspecto pacífico en cuanto a la producción de efectos negativos, los que no solo se despliegan a nivel individual, sino que también afectan esferas sociales y económicas (Medina Ariza, 2003). En este sentido, se ha considerado que este fenómeno corresponde a un problema en sí mismo, que genera un ciclo vicioso impactando en el bienestar de los individuos que, posteriormente, vuelve a retroalimentar los niveles de miedo (Jackson y Stafford, 2009, pp. 832 y 842) ${ }^{6}$. Así los efectos negativos son variados, entre los que se incluye la exclusión espacial y social que opera en diversas formas dependiendo de los grupos sociales afectados ${ }^{7}$.

En el marco de consecuencias que reviste el miedo al delito, resulta fundamental para efectos de este trabajo la instrumentalización política de la que puede ser objeto el miedo al delito, que tiene como resultado el devenir de prácticas populistas. La literatura comparada ha explorado el nexo entre populismo y miedo al delito, destacando su potencial político al ser capaz de demonizar a algunos sectores de la sociedad, intimidando y disciplinando al resto de la población que teme de ellos (Chevigny, 2003, p. 81). En este sentido, estudios empíricos han explorado la utilización del miedo al delito como arma política, confirmando la politización de algunos

\footnotetext{
${ }^{2}$ Con todo, los autores se cuestionan el significado de ciertas respuestas de los encuestados que podrían vincularse más bien al funcionamiento del sistema de justicia penal en general. Para una continuación de dicho análisis, véase Farrall (2004).

${ }^{3}$ En una reducción extrema, la metodología cualitativa puede ser caracterizada como un enfoque metodológico con orientación indagatoria, basado en la exploración y que opera mediante un razonamiento inductivo (Hernández Sampieri, Fernández Collado y Baptista Lucio, 2014, pp. 8 y 9). Con todo, se debe reconocer que la metodología cualitativa es una disciplina que abarca numerosos enfoques y métodos que atraviesan un complejo campo con diversos momentos históricos que operan en el presente. Al respecto, véase Denzin y Lincoln (2005, pp. 3 y ss.).

${ }^{4}$ Por ejemplo, véase la respuesta de una entrevistada: "Life shouldn't be like that, you shouldn't have to lock everything up and you know... once I've gone out, I'm not worried. But when I'm initially locking the door, it does make me angry".

${ }^{5}$ Así, se han realizado estudios que buscan determinar las correlaciones que existen entre el miedo al delito, ansiedad, depresión y otros factores relacionados con las actitudes en torno al delito.

${ }^{6}$ Esto se justificaría por el impacto que tiene en las personas y por la desconexión existente entre miedo y delito.

${ }^{7}$ Para una revisión pormenorizada de la literatura atendiendo a distintos grupos sociales considerando edad, género y otros, véase Pain (2000, pp. 372 y ss.).
} 
tópicos como crimen e inmigración vinculados al ascenso de grupos políticos de extrema derecha (Schuermans y De Maesschalck, 2010)․․

La realidad chilena no constituye una excepción a la tónica internacional descrita. Tal como ha apuntado la literatura chilena, ante la necesidad de implementar reformas, y frente a la ausencia de evidencia empírica para su justificación, se ha recurrido como argumento al temor que sentirían los ciudadanos (Morales Peillard, 2012). Es importante destacar que el "recurso" al miedo al delito no solo ha operado al momento de justificar cambios legislativos, sino que incluso se ha convertido en un horizonte programático contemplado desde ya en las propuestas de candidatos presidenciales. En este sentido, bastante ilustrativo resulta el comienzo del programa del presidente Sebastián Piñera en materia de seguridad ciudadana durante las elecciones del año 2017, el que señala:

"La seguridad ciudadana se está deteriorando en forma alarmante, lo que se expresa de distintas maneras. Muchas familias chilenas sienten inseguridady miedo en sus hogares, barrios, plazas y transporte público. La penetración del narcotráfico, las bandas y las pandillas han significado un serio deterioro en su calidad de vida. Junto a lo anterior, resultan especialmente graves las acciones terroristas en la zona sur del país. El retroceso de la seguridad ciudadana se ve reflejado en el alza de la victimización y el temor, indicadores que dan cuenta de un aumento de 228 mil hogares víctimas de la delincuencia durante el actual gobierno y un alza récord en la percepción de incremento del delito. Esta crisis nos ensombrece como sociedad y ocupa un lugar prominente entre las preocupaciones y temores de los ciudadanos" (Piñera Echeñique, 2017) ${ }^{9}$.

Esta amalgama entre política y miedo al delito ubica a la seguridad como un valor prioritario y autónomo dentro del sistema de justicia penal (Marquès i Banqué, 2017, p. 692), que se ha manifestado recientemente en la legislación chilena mediante la intensificación de las sanciones y en el otorgamiento de una mayor cantidad de

\footnotetext{
${ }^{8}$ Para una revisión dentro de zonas rurales y urbanas de Bélgica.

${ }^{9}$ Con todo, es importante destacar que el recurso al miedo al delito ha sido utilizado transversalmente por distintos sectores políticos para justificar la implementación de reformas político-criminales. Un ejemplo de esto es la "agenda corta" impulsada el año 2015 por la ex presidenta Michelle Bachelet. El mensaje presidencial de lo que finalmente conformó la Ley 20.931 (2016) indica: "El aumento de la actividad criminal y de la sensación de inseguridad por parte de la ciudadanía, medido a través de encuestas de victimización y tasas de denuncia, hacen necesario combatir los delitos de mayor connotación social".
} 
facultades intrusivas para el aparato estatal persecutor (Ley 20.931, 2016) ${ }^{10}$. La forma de ofrecer la justificación del miedo al delito para la adopción de medidas políticocriminales es mediante estadísticas donde se habla de "alzas" de percepción y temor. A continuación, se revisará esta la aproximación tradicional de medir el miedo al delito en el contexto comparado, con el fin de describir la forma en como se ha desarrollado la temática en Chile.

\section{La medición tradicional del miedo al delito: deficiencias metodo- lógicas}

Una somera revisión a la experiencia comparada revela que los principales instrumentos destinados a la medición del miedo al delito se encuentran conformados por la Crime Survey for England and Wales ${ }^{11}$, en tanto que la National Crime Victimization Survey constituye la principal herramienta de recolección de datos en Estados Unidos. Mientras que las encuestas de victimización en Estados Unidos utilizan la terminología de miedo al delito ("fear"), en Inglaterra y Wales se miden los niveles de preocupación ("worry") (Gray, Jackson y Farrall, 2010, p. 76). Pese a sus diferencias, es importante destacar que ambos instrumentos a lo largo del tiempo han afinado sus objetivos, pasando desde la mera contabilidad a medir niveles de miedo al delito, satisfacción con el aparato policial, entre otras cuestiones (Fattah, 2014, p. 8).

Tal como apunta Fattah (2014, p. 6), la utilización de encuestas conformó un "macro enfoque" que buscaba determinar el volumen de la población víctima y las características sociales, el que, sin embargo, no permitía "ahondar en las situaciones sociales y personales en donde acaecieron los delitos". La encuesta como recurso de elaboración de datos no solo ha constituido el paradigma sobre el cual se ha elaborado la información al miedo al delito, sino que también ha constituido la principal "técnica" con la que se ha investigado la subjetividad contenida en el miedo al delito (Narváez Mora, 2015, p. 104). Para efectos de este artículo, más allá de la cuestión sobre la encuesta como método para recopilar datos, lo relevante radica en el enfoque cuantitativo mediante el cual ha predominado la aproximación al miedo al delito ${ }^{12}$.

\footnotetext{
${ }^{10}$ Solo a modo de referencia, véase el aumento de penalidades y el control preventivo de identidad.

${ }^{11}$ Originariamente esta encuesta corresponde a la British Crime Survey, que mantuvo dicha denominación hasta el año 2012. El origen de la encuesta británica constituyó una respuesta del Home Office no solo para para recabar cifras negras, sino también para medir la percepción de riesgo e inseguridad en un contexto en que los partidos políticos de derecha en el Reino Unido abogaban por campañas vinculadas al slogan de la ley y el orden. Al respecto, véase, Gray, Jackson y Farrall, (2011, p. 3).

${ }^{12}$ En este sentido, las técnicas de investigación social se encuentran subordinadas al enfoque metodológico que se emplee. Para una revisión de las consecuencias que acarrea el optar por distintos enfoques, véase Bryman (2012, pp. 35 y ss.).
} 
Es importante destacar que la metodología cuantitativa busca la recolección de datos con el propósito de corroborar o refutar hipótesis, teniendo como base la medición numérica y el análisis estadístico (Hernández Sampieri, Fernández Collado y Baptista Lucio, 2014, p. 4). Este enfoque propone categorías numéricas/explicatorias, utilizando la medición como principal aproximación de análisis (Davis, 2016, pp. 4 y 5) ${ }^{13}$.

Si bien resulta innegable que la utilización de encuestas ha permitido recolectar información para sentar las bases sobre la discusión en torno al miedo al delito, estas han sido objeto de numerosas observaciones debido a la matriz metodológica bajo la cual operan. A tal punto se ha criticado la utilización de encuestas, que se ha llegado a denominárseles como las "archi-enemigas" del miedo al delito a propósito de la tergiversación que han producido en los niveles de miedo medido producto de su erróneo uso (Farrall, 2004, p. 158) ${ }^{14}$.

En primer lugar, se ha apuntado que las herramientas de índole cuantitativa no son las adecuadas para capturar respuestas emocionales que involucran el anger o fear of crime, al ser estas vagas, transitorias y fuertemente dependientes del contexto (Farrall, 2004, pp. 166-167). La operacionalización del miedo al delito mediante una metodología cuantitativa con una aproximación descontextualizada, a través de preguntas que se caracterizan por ser predefinidas y breves, ha provocado limitaciones tanto en el desarrollo conceptual como en la calidad de la información obtenida (Farrall, Bannister, Ditton y Gilchrist, 1997, p. 661). En este sentido, resulta relevante destacar que las encuestas de victimización terminan desatendiendo del carácter multifacético del fenómeno delictual (Farrall, Bannister, Ditton y Gilchrist, 1997, p. 662).

Vinculado con las dificultades descritas, las encuestas de victimización tienden a generar una descripción estática del delito, perdiéndose información relativa al proceso criminal, generando una descontextualización comparativamente mayor que la que ocurre en encuestas aplicadas a otras temáticas (Bowling, 1993, pp. 242243). En efecto, las experiencias y las variaciones emocionales vividas son difícilmente contenidas mediante la implementación de la encuesta de victimización (Farrall, Bannister, Ditton y Gilchrist, 1997, p. 660). Esto se ve aún más agudizado cuando las preguntas se formulan de manera dicotómica, impidiendo la identificación y diferen-

\footnotetext{
${ }^{13}$ Básicamente, los datos en el enfoque cuantitativo son números basados en el cálculo y enumeración. Una vez definido el problema de investigación, se construye el marco teórico del cual se extraen una serie de hipótesis que serán testeadas. Así, la investigación cuantitativa tiene una vocación generalizadora respecto de los resultados de la muestra, permitiendo tanto la explicación como la predicción de las relaciones causales entre los fenómenos estudiados mediante un razonamiento deductivo.

${ }^{14}$ Con todo, es importante destacar que este estrecho vínculo entre concepto, metodología y resultados obtenidos se da fuertemente en contextos similares, Para una revisión metodológica sobre actitudes punitivas, (Hutton, 2005), cómo es la medición de las actitudes punitivas por parte de los ciudadanos ante el castigo.
} 
ciación de frecuencia e intensidad en la medición del miedo al delito (Lim y Chun, 2015, p. 142).

A nivel empírico, numerosos estudios han dado cuenta del diverso impacto que tiene la utilización de preguntas abiertas en oposición a preguntas cerradas, las que terminan sobreestimando los niveles de miedo al delito (Farrall, Bannister, Ditton y Gilchrist, 1997, pp. 661-662). Ejemplo de estas formulaciones pueden encontrarse inclusive dentro de propuestas en la literatura castellana reciente, donde preguntas relativas a la inseguridad y temor se formulan en términos positivos ${ }^{15}$. De esta mane$\mathrm{ra}$, se termina sometiendo a un ejercicio inductivo a los entrevistados, donde son llevados a justificar estados de ánimo en base a juicios que carecen de reflexión alguna (Cornelli, 2008, p. 107).

Pasando desde del ámbito de la formulación de preguntas al de respuestas, la literatura comparada también ha detectado otro problema asociado al formato de preguntas: la interpretación que realizan los entrevistados de estas. En algunos casos, las preguntas son interpretadas en términos hipotéticos por parte de los encuestados, impactando en los datos que se construyen finalmente. Dentro de las entrevistas cuantitativas esto plantea una dificultad mayor, ya que el encuestador no puede corroborar si una respuesta se encuentra evaluando reacciones existentes o hipotéticas (Farrall, Bannister, Ditton y Gilchrist, 1997, p. 666). Por ejemplo, dentro de una encuesta en el marco de una investigación, una entrevistada manifestó el máximo nivel de preocupación ante la pregunta de cuan preocupada está de que alguien cometa actos vandálicos dentro de su casa o en los alrededores. Posteriormente la misma persona en una entrevista cualitativa sostuvo que "me preocuparía si me ocurriera", marcando un nivel de preocupación completamente distinto al manifestado anteriormente (Farrall, Bannister, Ditton y Gilchrist, 1997, pp. 666-667).

En esta misma línea de reflexiones sobre la forma de investigar el miedo al delito, destaca un reciente meta-análisis en la materia, mediante el cual se comprobó el impacto que tiene en las conclusiones el diseño de los estudios (Collins, 2016, p. 27). En dicha investigación se dio cuenta, entre otras cuestiones, de las diferencias que provoca la formulación de preguntas sutilmente distintas sobre el miedo a caminar en el vecindario (Collins, 2016, p. 28).

\footnotetext{
${ }^{15} \mathrm{Al}$ respecto, la propuesta de instrumento de Fernández Molina y Grijalva Eternod (2012, p. 11) incluye preguntas del siguiente tenor: "MDA1: En relación con la delincuencia, ¿consideras que vivir en el D.F. es inseguro?; MDA2: En relación con la delincuencia, ¿consideras que vivir en tu colonia es inseguro?; MDA3: En relación con la delincuencia, ¿sientes temor al caminar por tu colonia?; MDA4: En relación con la delincuencia, ¿sientes temor al estar solo/a en casa?".
} 


\section{La medición del miedo al delito en Chile}

\subsection{Debate conceptual}

En el entendido de que el primer paso para selección de la metodología- y posteriormente el método- más idóneo para la investigación de un fenómeno es la discusión de su concepto, se procederá a reseñar el estado actual de la discusión en Chile. Este debate ha sido acotado, salvo contadas excepciones en el que el foco ha estado puesto más bien en la relevancia política del fenómeno producto de las altas cifras arrojadas por las encuestas de medición (Morales Peillard, 2012, pp. 103 y ss.) ${ }^{16}$. Es así como ya desde antes del inicio del presente siglo investigaciones cuantitativas en el medio chileno arrojaban que las cifras de inseguridad no guardaban relación con las tasas de victimización (Oviedo y Rodríguez, 1999, p. 283).

Dentro de los trabajos que se han pronunciado sobre el aparato teórico detrás del miedo al delito, destaca la contribución de las investigadoras Dammert Guardia y Malone (2003, p. 79), ambas autoras se cuestionaron las razones que explicarían por qué en Chile existían a la fecha cifras tan altas de miedo al delito a pesar de ser un país con bajas tasas de criminalidad. Literalmente, las autoras se cuestionaron: "¿Por qué los chilenos tienen tanto miedo si su país es tan seguro?"17.

Dammert Guardia y Malone (2003, p. 80) adoptaron la tesis de que el miedo al delito en Chile debe ser explicado por otros factores ajenos al delito, vinculados a aspectos económicos, sociales y políticos presentes en nuestro país (Dammert Guardia y Malone 2006, p. 46). Así, optaron por la creación de una escala de inseguridad, operacionalizando tanto el miedo sobre violencia general como el miedo de violencia al asalto o robo (Dammert Guardia y Malone, 2003, p. 84). Las autoras enfocaron su estudio en el aspecto afectivo del miedo al delito- tanto en su vertiente abstracta como concreta- corroborando finalmente la hipótesis de que el miedo al delito es afectado por un contexto de inseguridades que incluyen las tasas de desempleo y pobreza (Dammert Guardia y Malone, 2003, pp. 70 y 95). Es importante destacar que la tesis de las autoras ya había sido esbozada con anterioridad, e incluso vigente hoy en día con estudios que contemplan dentro de los factores explicativos categorías como el nivel de protección que se ostenta ante desastres naturales o en temas de derechos humanos (Garcia, Francisco y Caliso, 2017, p. 22).

A pesar del innegable impacto que ha tenido la investigación de Dammert Guardia y Malone dentro de los estudios sobre el miedo al delito, este no se encuen-

\footnotetext{
${ }^{16}$ La delincuencia comenzó a ser abordada como un tema político a mediados de los años noventa en Chile. Para una revisión del recurso a la inseguridad subjetiva como argumento para justificar medidas punitivas dentro de las agendas políticas.

${ }^{17}$ La paradoja sobre baja victimización y alto miedo al delito en ciudades consideradas como seguras también ha impulsado la realización de otras investigaciones recientes en la materia. Al respecto, véase el ejemplo de Barcelona en Valera Pertegàs, S. y Guàrdia Olmos (2014).
}

Rev. derecho (Coquimbo, En línea) 2019, 26: e3860 
tra exento de problemas metodológicos que se hallan en estricta correlación con las asunciones conceptuales descritas. El principal cuestionamiento radica en la asunción por parte de las investigadoras de que las cifras arrojadas como miedo al delito corresponden efectivamente a dicho fenómeno, y no a otro constructo tomando en consideración la forma en cómo se han elaborado los datos en Chile. Las autoras parten de la base- y luego corroboran mediante su estudio- la existencia de un alto nivel de miedo al delito en Chile, buscando una explicación de la clásica paradoja entre baja victimización/alto miedo sin cuestionarse la existencia de ella considerando el enfoque mediante el cual se han obtenido dichos datos. De esta manera, resulta difícil la diferenciación entre medición y concepto, ya que el primero estaría en todo caso limitando desde su raíz la definición de los contornos del segundo.

Paralelamente a la contribución de Dammert Guardia y Malone, Medina Ariza (2003) publicó en España un artículo precisamente en el sentido contrario, criticando la noción de inseguridad ciudadana, donde sostiene que la noción del miedo al delito es menos genérica y ambigua, encontrando un mayor desarrollo dentro de la literatura. Además de dichas razones, este artículo sostiene la necesidad de estudiar el fenómeno desde esta perspectiva reducida considerando las implicancias que tiene la identificación de las medidas que puede adoptar la Política Criminal ante dicho fenómeno.

La adopción de un concepto circunscrito al fenómeno criminal que excluya las ansiedades propias del desarrollo económico y social, y a su vez multidimensional- englobando aspectos emocionales, cognitivos y conductuales- puede resultar útil para la construcción de información empírica idónea para la toma de decisiones sobre políticas públicas en este ámbito. En este sentido, la noción de inseguridad descrita anteriormente no resulta operativa para dichos efectos, siendo difícil detectar las medidas que se pueden adoptar desde los estudios de la Política Criminal ante la necesidad de, por ejemplo, crear empleos más estables para combatir el miedo al delito. En otras palabras, se sostiene que los datos recopilados por medio del concepto de inseguridad ciudadana no solo no constituyen conceptualmente "miedo al delito", sino que, al final del día, su asunción no posibilita la construcción de datos que permitan informar políticas focalizadas para reducir las causas que provocarían los niveles de temor. Lo anterior parte de la base de que una política de seguridad urbana completa va más allá del tratamiento de la delincuencia, requiriendo llevar a cabo medidas para disminuir los niveles del miedo al delito (Barberet, 2004, p. 169).

\subsection{Metodología y medición del miedo al delito en Chile}

A nivel empírico, los principales instrumentos con aplicación periódica en Chile que proveen de datos sobre criminalidad, violencia e inseguridad en nuestro país se encuentran constituidos por la Encuesta Nacional Urbana de Seguridad Ciudadana (en adelante "ENUSC") (Subsecretaría de prevención del Delito del Ministerio del Inte- 
rior y Seguridad Pública, 2013) ${ }^{18}$ junto con el Índice-Paz Ciudadana-GFK Adimark (en adelante "IPCD") (Dammert Guardia, Karmy Bolton y Manzano Chávez, 2005, p. 13 ; Fuentealba Carrasco, Rojas González y Barriga, 2016) ${ }^{19}$. Tanto la ENUSC como el IPCD se enmarcan dentro la tendencia comparada ya reseñada en el acápite anterior, distinguiéndose por ser encuestas que buscan contabilizar y caracterizar el fenómeno.

A pesar de la innegable contribución que estos instrumentos han realizado a la caracterización del tópico a nivel local, existen diversas dimensiones del miedo al delito que no pueden ser estudiadas mediante el enfoque cuantitativo adoptado (San Martín, 2013, p. 75 y Dammert Guardia, Karmy Bolton y Manzano Chávez, 2005, p. 13). En particular, se sostiene que el trabajo empírico chileno se ha orientado mayoritariamente hacia la descripción del fenómeno, dejándose en segundo plano objetivos fundamentales de la investigación social, como en este caso lo son la exploración y explicación del miedo al delito ${ }^{20}$. Para fundamentar lo anterior, se revisarán los instrumentos en el marco de los problemas propios de la metodología cuantitativa ya reseñados, atendiendo también a ciertas particularidades que reviste la aplicación de estas encuestas.

\subsection{1. Índice Paz Ciudadana-Gfk Adimark}

El IPCD es una encuesta semestral que construye la información mediante una metodología cuantitativa mixta, recurriendo tanto a encuestas telefónicas como personales para el levantamiento de datos. La disyuntiva sobre aplicar una encuesta presencialmente o vía telefónica en el ámbito del miedo al delito ya ha sido discutida con anterioridad por parte de la literatura comparada (Skogan, 1999, p. 40) ${ }^{21}$. El cuestionamiento aquí sostenido, sin embargo, dice relación con la utilización conjunta de formularios aplicados vía telefónica y personal dentro de un mismo estudio sobre miedo al delito.

Como ya se ha explicado en el apartado sobre deficiencias metodológicas, la medición del miedo al delito es una materia particularmente sensible al formato y al contenido de las preguntas con que se construyen los datos. Si bien la publicitación de los resultados de IPCD transparentan desde un inicio las diversas modalidades de recopilación de información, esto necesariamente tiene un impacto en los resultados al generarse la producción de datos en contextos distintos, como lo son las variantes

\footnotetext{
${ }^{18}$ El sitio web gubernamental indica: "La Encuesta Nacional Urbana de Seguridad Ciudadana (ENUSC) es el instrumento más relevante en el país para indagar en torno a las consecuencias de la criminalidad, violencia e inseguridad".

${ }^{19}$ En esta línea, dentro de los últimos estudios llevados a cabo en nuestro país, destaca la investigación sobre percepción de inseguridad tanto a nivel cognitivo como emocional en el Gran Concepción.

${ }^{20}$ Sobre los fines de la investigación social, véase Babbie (1996, pp. 113 y ss.).

${ }^{21}$ Así, para este autor, el debate estaría enfocado en los costos y beneficios que implica cada técnica. Mientras que la encuesta presencial resulta más costosa y plantea dificultades de acceso al hogar del encuestado, la encuesta telefónica en las ciudades sin acceso a este recurso plantearía problemas de muestreo.
} 
de encuestas con y sin presencia física de los encuestadores. Al optarse por una metodología mixta que incluye recogida de información vía telefónica, es necesario tener en consideración el efecto conocido como "ausencia de diferenciación" que se produce en este formato de encuestas a distancia. Así, en este tipo de instrumentos se generan mayores dificultades para incentivar al encuestado junto a una mayor urgencia por finalizar el ejercicio, lo que finalmente deviene en que "el entrevistado realiza una valoración del primer aspecto y — una vez elegida una respuesta-la repite a lo largo de todos los ítems; en vez de llevar a cabo un razonamiento cognitivo en cada uno" (Díaz de Rada Igúzquiza, 2010, p. 101-102) ${ }^{22}$. Esto es especialmente relevante a efectos de las encuestas del miedo al delito, donde la complejidad del fenómeno lleva a la realización de preguntas sutilmente diversas de acuerdo a las distintas aristas que se estén analizando.

Relacionado con lo anterior, el IPDC construye un indicador global que abarca tanto aspectos cognitivos como emocionales del miedo al delito, operando a su vez con preguntas formuladas en términos abstractos sumado a la consulta del robo en particular (Fundación Paz Ciudadana y GFK Adimark, 2017) ${ }^{23}$. Dependiendo de las respuestas que entregan los entrevistados, estos se agrupan en las categorías de temor bajo, medio y alto. Los últimos resultados de la encuesta destacan que el nivel de personas que se encuentran en el índice de temor alto disminuyó sin magnitudes significativas de un $17.9 \%$ en octubre del año 2016 a un $16.6 \%$ en septiembre de 2017. Lo anterior no es sino una continuación de la tendencia a la baja experimentada entre los años 2015 y 2016 (Fundación Paz Ciudadana y GFK Adimark, 2016)24.

En concordancia con la disminución del sector de la población que se encuentra en un nivel de temor alto, en el plano conductual también se registra una baja estadísticamente significativa. Así, el porcentaje de personas que afirma haber reforzado la seguridad de su casa disminuye de un $64.8 \%$ en 2015 a un $56.5 \%$ en comparación con la encuesta anterior ${ }^{25}$.

Uno de los datos más interesantes dice relación con la diferenciación entre hogares víctima (de robo o intento de robo dentro de los últimos seis meses) y hoga-

\footnotetext{
${ }^{22}$ Esto se vincula con la posibilidad de obtener mayores respuestas afirmativas. A modo de referencia, véase las variaciones porcentuales entre encuestas telefónicas y presenciales en una materia que también busca la medición de percepciones subjetivas como lo es el voto político.

${ }^{23}$ Estas variables corresponden a: percepción de la variación en la violencia de la delincuencia en su comuna, proyección de la variación de la delincuencia en su comuna en el futuro, temor de ser asaltado cuando sale de su casa a trabajar, temor de ser asaltado cuando vuelve al anochecer a su casa, temor de ser asaltado al caminar solo por su barrio y temor de ser asaltado dentro de su casa por las noches.

${ }^{24}$ Para una revisión sobre las variaciones porcentuales diferenciadas por segmento económico, y Santiago/regiones

${ }^{25}$ Los demás ítems relativos se mantienen sin variación significativa. Estos corresponden a: ponerse de acuerdo con vecinos para ayudarse; dejar de salir a ciertas horas y dejar de ir a ciertos lugares.
} 
res no víctima. Mientras el primer grupo manifiesta un $25.8 \%$ de temor alto, los hogares no víctima se reducen a menos de la mitad con un $10.5 \%$.

Considerando los datos recientemente descritos, resulta posible señalar que la victimización- focalizada en la comparación de hogares- corresponde a un factor relevante al momento de buscar las causas vinculadas a los niveles de miedo al delito. A nivel conductual, si bien la información permite ilustrar sobre las variaciones porcentuales con y sin significancia estadística, no permite establecer correlaciones que permitan determinar las razones que influyen en las variaciones, como tampoco posibilita la exploración sobre eventuales nuevas prácticas llevadas a cabo por las personas no contempladas por el instrumento. Estas limitaciones, por cierto, forman parte de las restricciones propias de la entrevista cuantitativa, donde se reducen las posibilidades y espacios de profundizar respuestas al impedir la narración de experiencias por parte del entrevistado.

\subsubsection{Encuesta nacional urbana de seguridad ciudadana}

La ENUSC corresponde a un instrumento con aplicación anual desde el año 2003, estructurado en torno a la realización de entrevistas personales. Mediante las preguntas iniciales la encuesta busca determinar el posicionamiento que ostenta para los ciudadanos la temática de la delincuencia- y del miedo al delito en particular- en el marco de los grandes tópicos de relevancia nacional. No resulta menor, sin embargo, que ante las pregunta sobre problemas de relevancia nacional se formulen como distintas posibilidades de respuesta la delincuencia, el tráfico de drogas y la corrupción. Esto no viene sino a configurar un concepto de delincuencia diverso a las conductas relacionadas al tráfico de drogas y corrupción, pudiendo desprenderse de la lectura completa del instrumento que la noción de delincuencia se encuentra asociada más bien a los delitos contra la propiedad ${ }^{26}$.

Una somera revisión de la encuesta permite detectar la presencia de varias preguntas focalizadas en la noción de incivilidades. Así, hacia el inicio de la encuesta se pregunta por las "incivilidades" 27 y "violencias" 28 que los encuestados perciben en su barrio, mezclando de paso conductas tipificadas y no tipificadas por el ordena-

\footnotetext{
${ }^{26}$ Así, es necesario considerar que la encuesta toma como base para la descripción y análisis de datos los delitos de robo con violencia o intimidación, robo por sorpresa, hurto y lesiones. Con todo, no se desconocen los esfuerzos detrás de la encuesta por consultar hacia el final de la misma sobre victimización de delitos de daños, connotación económica, cibernéticos y amenazas.

27 En la ENUSC las incivilidades abarcan una diversidad de conductas, que van desde Prostitución/Comercio sexual, venta clandestina de alcohol, comercio ambulante, personas durmiendo en la calle/pidiendo dinero, rayados, sitios eriazos descuidados/acumulación (...) Consumo alcohol/droga en la vía pública, perros abandonados.

${ }^{28}$ Entre ellas se encuentran: amenazas o peleas entre vecinos, haber presenciado peleas callejeras sin armas, haber presenciado peleas callejeras con armas, presencia de pandillas violentas, robos o asaltos en la vía pública, vandalismo, escuchar balaceras o disparos, venta de drogas.
}

Rev. derecho (Coquimbo, En línea) 2019, 26: e3860 
miento normativo chileno ${ }^{29}$. Al respecto, la encuesta acierta al consultar mediante formulaciones separadas cuales serían las causas de la delincuencia y cuáles son las medidas que combatirían la inseguridad. Es importante destacar que a nivel de publicitación de resultados estos datos no se encuentran disponibles en las páginas de consulta, lo que impide una divulgación de datos esenciales para el diseño de medidas que consideren las respuestas de los encuestados (Barberet (2000, p.234) ${ }^{30}$.

Dentro de los resultados publicitados se destacan bajas en los niveles de miedo en el año 2016 en comparación al 2015. Así, disminuye de un $86.8 \%$ a un $85.0 \%$ las personas que perciben que la delincuencia aumentó. En la misma línea, varía de un 41.3\% a 39.7\% la población que cree que será víctima de un delito en los próximos doce meses. Dichos porcentajes van en línea con las tendencias a la baja descritas a través del IPCD. En relación a la victimización para explicar el miedo al delito, tal como ha advertido con anterioridad la literatura chilena, no resulta posible detectar un "flujo de relación" entre niveles de delincuencia y percepción (San Martín, 2013, p. 74). Así, mientras la victimización personal es de un $9.6 \%$ y la de hogares de un $27.3 \%$, las personas que perciben que la delincuencia aumentó corresponden al $85 \%$ y el $39.7 \%$ cree que será víctima de un delito.

Por otro lado, la ENUSC expone las fuentes de información utilizadas por las personas para realizar sus propios juicios sobre la percepción de aumento en la delincuencia. En el mismo ciclo analizado, se destaca una reducción de un $69.7 \%$ a un $64.9 \%$ en relación a los medios de comunicación (TV, radios, prensa escrita), a la vez que detecta un aumento de un $30.0 \%$ a un $34.4 \%$ sobre los que se informan vía experiencia personal y de otras personas. En suma, habría un incremento del conocimiento que se obtiene mediante experiencias relativas a la victimización primaria y secundaria. Con todo, si bien los datos describen de las prácticas sobre cómo se informan los chilenos, no permiten determinar una eventual influencia de estos en la reacción de temor, por lo menos a nivel de publicitación de datos ${ }^{31}$.

\footnotetext{
${ }^{29}$ De acuerdo a esta teoría, las conductas vinculadas a incivilidades y desórdenes dan cuenta de la ausencia de control y preocupación (por parte del resto de la comunidad). Partiendo de la base que el control brinda seguridad, la ausencia de este generaría mayores niveles de miedo al delito. Al respecto, Farrall, Gray y Jackson (2007, pp. 8 y ss.).

${ }^{30}$ Los estudios criminológicos han sido enfáticos en la necesidad de divulgar los datos que se construyen en las investigaciones.

${ }^{31}$ Dentro de las investigaciones chilenas que han estudiado el vínculo entre consumo de noticias y temor en Chile, destaca la contribución de Scherman Teilerboim y Etchegaray Thielemann (2013, pp. 567,573 y 574). En particular, se concluyó que el único medio con relevancia positiva es la televisión, presentando una influencia menor que otros factores como edad o victimización previa. Con todo, esta investigación se remite a la faceta de miedo al delito afectiva.
} 


\section{Sugerencias para futuras investigaciones sobre miedo al delito en Chile}

A la luz de las dificultades identificadas, resulta necesario volver al punto inicial de toda investigación: ¿Cuál es el objetivo de la investigación? La correcta identificación del objetivo y su traducción en pregunta de investigación permiten establecer la metodología y posteriormente los métodos más adecuados para construir la información buscada. La escasa precisión de esta interrogante en esta materia ya ha sido advertida por Fattah $(2014$, p. 14) quien ha retornado a la pregunta base: "¿Qué tratan de medir exactamente las encuestas de victimización?". En el contexto chileno descrito, se concluye que la producción de conocimiento social sobre el miedo al delito- basado mayoritariamente en un enfoque cuantitativo- se ha concentrado principalmente en su dimensión descriptiva, dejando de lado otros objetivos de la investigación científica como lo son la exploración y explicación del fenómeno. En este sentido, las futuras investigaciones debiesen poner un mayor énfasis en estos últimos dos niveles mediante la inclusión de metodologías mixtas por medio de la utilización de métodos cualitativos de investigación social ${ }^{32}$. Si bien no se desconoce la utilización de esta metodología en los estudios chilenos, estos se han orientado y justificado en base a fines diversos a los aquí propuestos (Dammert Guardia, Karmy Bolton y Manzano Chávez, 2005, pp. 11-14) ${ }^{33}$.

Lo anterior debe comprenderse bajo el entendido que la elección de la metodología empleada no es una decisión azarosa o inocente. En este sentido, la forma de producir la información relativa al miedo al delito en Chile ha resultado funcional para los propósitos populistas con los cuales se ha promovido en el mundo político. La generación de cifras sin una perspectiva definida sobre qué se está midiendo ha devenido en una instrumentalización del fenómeno en vez de promover una discusión sobre sus límites, causas y real existencia de éste.

A nivel exploratorio, se sugiere incursionar en dimensiones del fenómeno que no han sido investigadas en Chile, como lo es la noción de anger of crime. Lo anterior no solo viene a llenar una necesidad de explorar aspectos que no han sido tratados en el contexto chileno, sino que surge de necesidades ya resaltadas por la investigación empírica local. Así, estudios recientes en el medio chileno han concluido que un mismo delito provoca distintos niveles de temor tanto a nivel cognitivo como emocional, lo que daría cuenta de las distintas experiencias subjetivas que se generan dentro de los individuos (Fuentealba Carrasco, Rojas González y Barriga, 2016, pp. 644-645). La confirmación de la diversidad contenida en la experiencia en Chile sobre

\footnotetext{
${ }^{32}$ La aplicación de métodos cualitativos ha tenido una interesante tradición y evolución dentro de los estudios criminológicos que exceden el tópico del miedo al delito. Para una revisión del ejercicio comparativo sobre la utilización de ambos enfoques en estudios sobre bandas juveniles entre Sampson/Laub y Jankowski, véase Corbetta (2007, pp. 32-40).

${ }_{33}$ Por ejemplo, se ha buscado explorar el vínculo entre democracia, espacio público y ciudadanía. Rev. derecho (Coquimbo, En línea) 2019, 26: e3860
} 
reacciones frente al delito da cuenta de la necesidad de precisar y evaluar dichas reacciones.

En concordancia con lo expuesto, se requiere la inclusión de nuevos focos de análisis, siendo pertinente, por ejemplo, la utilización de perspectivas de género en el caso de ilícitos explorados solo tangencialmente en el contexto chileno como lo es el miedo frente a los delitos sexuales (Lorenc, et al., 2013) ${ }^{34}$. En este sentido, se requiere también la aplicación de estudios focalizados a poblaciones particulares, bajo la comprensión de que ostentan dinámicas y necesidades propias como la población universitaria (Barberet y Fisher, 2009) o el conformado por adultos mayores ${ }^{35}$.

Es importante destacar que la demanda de complementar la metodología cuantitativa con recursos cualitativos ya ha sido tempranamente detectada por estudios comparados, los que han sido llevados a cabo en búsqueda de una aproximación metodológica pluralista (Farrall, Bannister, Ditton y Gilchrist, 1997, p. 662) ${ }^{36}$. En este sentido, investigaciones comparadas han combinado metodologías de orden cuantitativo y cualitativo, en donde los investigadores luego de implementar una serie de encuestas, han vuelto a aplicar entrevistas semiestructuradas sobre los mismos participantes. Los distintos resultados obtenidos "mismatch" son atribuidos por parte de los autores directamente a la metodología empleada, descartando la existencia de diferencias producto de cambios de actitudes o emociones (Farrall, Bannister, Ditton y Gilchrist, 1997, p. 664) ${ }^{37}$. En ese sentido, una de las conclusiones del estudio más relevantes para este documento es que la metodología cuantitativa ha continuamente sobreestimado los niveles de miedo al delito (Farrall, Bannister, Ditton y Gilchrist, 1997, p. 676).

Dentro de este complemento por parte de metodologías cualitativas que vayan en paralelo a los estudios cuantitativos ${ }^{38}$, se sugiere que se incluyan en futuras investigaciones preguntas en las cuales se apunte a que el encuestado rememore un hecho- dentro del período investigado - que le cause miedo/enojo relativo a la victimización ${ }^{39}$. Esto permite construir una visión más acabada del fenómeno, otorgando

\footnotetext{
${ }^{34}$ En este sentido, en una interesante revisión sistemática de estudios cualitativos sobre miedo al delito se concluyó que la perspectiva de género corresponde a la dimensión más relevante.

${ }^{35}$ Para la revisión de un estudio sobre espacio público, miedo al delito y adultos mayores, véase, Ceccato y Bamzar (2016, pp. 115-133).

${ }^{36}$ Así, con particular énfasis en el estudio de casos, Bowling (1993, pp. 244-246).

37 Esto no es sino una expresión particular de los efectos que se producen luego de la adopción de distintas metodologías sociales. Tal como apuntan Pole y Lampard (2002, p. 4). usando distintas aproximaciones en la investigación y recolectando distintos tipos de datos, podremos decir distintos tipos de cosas, dirigir distintos tipos de preguntas y producir distintos resultados de la investigación.

$38 \mathrm{Si}$ bien no se desconoce la existencia de estudios cualitativos sobre el miedo al delito en nuestro medio, estos han estado orientado a distintas finalidades. Al respecto, véase San Martín (2013); Dammert Guardia, Karmy Bolton y Manzano Chávez (2005, p. 13).

${ }^{39}$ Así, similarmente como se utiliza en Farrall (2004, p. 167).
} 
un espacio para la reflexividad y profundización de detalles que el entrevistado considere importante en torno al miedo al delito.

La necesidad de incluir preguntas directas y específicas relativas al origen del temor se vincula con el segundo de los puntos en los cuales la investigación empírica debiese profundizar: las causas del miedo al delito en Chile. Parte de la profundización de datos implica identificar las causas que motivan o reducen los niveles de

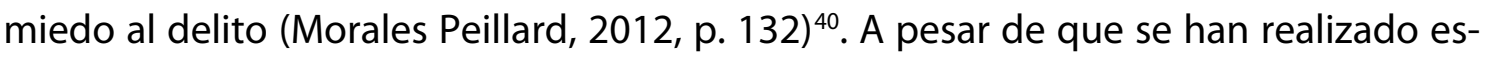

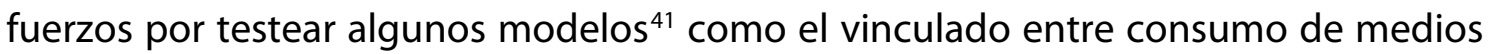
de comunicación, el rechazo a la tesis de la victimización o control social, estos se han medido fragmentariamente, siendo difícil comparar resultados o realizar un eventual meta-análisis debido a la diversidad de técnicas y conceptos empleados.

Reconociendo que ninguna metodología es capaz de abarcar todas las dimensiones del fenómeno (Gray, Jackson y Farrall, 2011, p. 12), las sugerencias tanto a nivel cuantitativo como cualitativo planteadas se orientan bajo una misma directriz: construir información que resulte idónea para la implementación de medidas que se puedan ejecutar desde la Política Criminal para reducir los niveles de miedo al delito ${ }^{42}$. Así, se busca que el fenómeno del miedo al delito comience a desligarse de su función prioritariamente justificante en el endurecimiento de medidas penales, abriendo paso a discusiones y narrativas más reflexivas mediante técnicas de investigación cualitativa.

Un paso fundamental para la inclusión de esta perspectiva cualitativa es el reconocimiento de la relevancia de la subjetividad contenida en las experiencias de las personas. Esto encuentra correlato por lo apuntado en la literatura, la cual sostiene que "para entender el procesamiento de los datos que entrega la percepción para la toma de decisiones, también es necesario considerar estos sesgos" (Piña Rochefort, 2012, pp. 125-126). En efecto, resulta posible considerar estas variaciones subjetivas a efectos de implementar políticas vinculadas a la reducción de las percepciones sobre miedo al delito, siempre que se esté tratando este fenómeno y no otro tipo de inseguridades inherentes a la convivencia en el mundo contemporáneo.

\footnotetext{
${ }^{40}$ Sugiere también la búsqueda de modelos teóricos adicionales para explicar las tasas de miedo al delito en nuestro país.

${ }^{41}$ Dentro de los esbozos teóricos más recientes en el campo, es posible destacar la contribución proveniente desde el mundo de la sicología denominada construal level theory. Según esta teoría existen dos tipos de constructos mentales, asociados a la abstracción/contextualización (nivel alto) o constructo concreto, subordinado y contextual (nivel bajo). Este modelo favorece la aplicación de metodologías cualitativas, por lo que su exploración puede resultar útil conforme a las sugerencias ya descritas en el documento. Al respecto, véase Gouseti y Jackson (2015, pp. 3 y ss).

${ }^{42}$ En este sentido, resulta necesario destacar que la producción de conocimiento válido corresponde al objetivo planteado. El diseño de las políticas para tratar la problemática corresponde a un paso posterior. Sobre dicha confusión, véase Wainerman y Sautu (2011, pp. 28 y 29).
}

Rev. derecho (Coquimbo, En línea) 2019, 26: e3860 
Déficits en la medición del miedo al delito en Chile.

\section{Agradecimientos}

Una versión preliminar de este artículo fue expuesto en las XIV Jornadas Chilenas de Derecho Penal y Ciencias Penales, organizadas por la Universidad Católica del Norte en conjunto con la Universidad de Antofagasta durante noviembre 2017. Agradezco a los profesores Francisco Maldonado y Sebastián Salinero por la paciencia empleada para revisar y comentar pormenorizadamente un borrador de este manuscrito.

\section{Referencias Bibliográficas}

Babbie, E. R. (1996). Manual para la práctica de la investigación social. Bilbao: Desclée de Brouwer.

Barberet, R. (2000). La investigación criminológica y la política criminal. Revista de derecho penal y criminología, (5), 221-242. Recuperado de https://bit.ly/35zHfcS

Barberet, R. (2004). La seguridad urbana: la experiencia europea y las consecuencias para América Latina. En J. L. Rhi-Sausi (Ed.). El desarrollo local en América Latina. Logros y desafíos para la cooperación europea (pp. 163-176). Caracas: Recal-CesSPI.

Barberet, R. y Fisher, B. S. (2009). Can security beget insecurity? Security and crime prevention awareness and fear of burglary among university students in the East Midlands. Security journal, 22(1), 3-23. https://doi.org/10.1057/sj.2008.9.

Bowling, B. (1993). Racial harassment and the process of victimization: conceptual and methodological implications for the local crime survey. The British journal of criminology, 33(2), 231-250. Recuperado de https://bit.ly/2rwuDEG

Bryman, A. (2012). Social research methods (4a ed.). Oxford: Oxford University Press.

Canare, T, Francisco, J. P. y Jopson, E. M. (2017). Fear of crime and saving behavior. RSN-PCC Working Paper ,1 7(002), 1-33. https://doi.org/10.2139/ssrn.2906621.

Ceccato, V. y Bamzar, R. (2016). Elderly victimization and fear of crime in public spaces. International criminal justice review, 26(2), 115-133. https://doi.org/10.1177/1057567716639096.

Chevigny, P. (2003). The populism of fear: Politics of crime in the Americas. Punishment \& Society, 5(1), 77-96. https://doi.org/10.1177/1462474503005001293.

Collins, R. (2016). Addressing the inconsistencies in fear of crime research: a meta-analytic review. Journal of criminal justice, 47, 21-31. https://doi.org/10.1016/j.jcrimjus.2016.06.004. 
Corbetta, P. (2007). Metodología y técnicas de investigación social. (C. Fraile Maldonado \& M. Fraile Maldonado, Trads.) Madrid: McGraw Hill.

Cornelli, R. (2008). Miedo, criminalidad y orden. (F. Valgiusti, Trad.). Buenos Aires: B de F.

Dammert Guardia, L, Karmy Bolton, R. y Manzano Chávez, L. (2005). Ciudadanía, espacio público y temor en Chile. Santiago: CESC. Recuperado de https://bit.ly/2Os2EzO

Dammert Guardia, L. y Malone, M. F. T. (2003). Fear of crime or fear of life? Public insecurities in Chile.Bulletin of latin american research,22(1), 79-101. https://doi.org/10.1111/1470-9856.00065.

Dammert Guardia, L. y Malone, M. F. T. (2006). Does it take a village? Policing strategies and fear of crime in Latin America. Latin American politics and society, 48(4), 2751. Recuperado de https://bit.ly/2KZA31X

Davis, K. (2016). Qualitative and quantitative research methods and rhetorical theory in the 'third generation'. En Proceedings of the Legal writing institute biennial conference. Scholarship: Who are we now? legal writing scholarship in today's academy. Portland, Oregon. Recuperado de https://bit.ly/2QU6kLD

Denzin, N. y Lincoln, Y. (2005). The discipline and practice of qualitative research. En su Handbook of qualitative research (pp. 1-19). London: Sag. Recuperado de https://bit.ly/2OSErkm

Díaz de Rada Igúzquiza, V. (2010). Comparación entre los resultados proporcionados por encuestas telefónicas y personales: el caso de un estudio electoral. Madrid: CIS. Recuperado de https://bit.ly/35EgWCl

Ditton, J., Bannister, J., Gilchrist, E. y Farrall, S. (1999). Afraid or angry? recalibrating the 'fear' of crime. International review of victimology, 6(2), 83-99. https://doi.org/10.1177/026975809900600201.

Ditton, J., Farrall, S., Bannister, J., Gilchrist, E. y Pease, K. (1999). Reactions to victimisation: why has anger been ignored? Crime prevention and community safety, 1(3), 3754. https://doi.org/10.1057/palgrave.cpcs.8140024.

Farrall, S., Bannister, J., Ditton, J., y Gilchrist, E. (1997). Questioning the measurement of the fear of crime: findings from a major methodological study. British journal of criminology, 37(4), 658-679. https://doi.org/10.1093/oxfordjournals.bjc.a014203.

Farrall, S. (2004). Revisiting crime surveys: emotional responses without emotions? OR Look back at anger. International journal of social research methodology, 7(2), 157171. https://doi.org/10.1080/1304557021000024767. 
Farrall, S., Gray, E. y Jackson, J. (2007). Theorising the fear of crime: the cultural and social significance of insecurities about crime.SSRN electronic journal. https://doi.org/10.2139/ssrn.1012393.

Farrall, S., Jackson, J. y Gray, E. (2009). Social order and the fear of crime in contemporary times. Oxford: Oxford University Press. https://doi.org/10.1093/acprof:oso/9780199540815.001.0001.

Fattah, E. (2014). Victimología: pasado, presente y futuro. Revista electrónica de ciencia penal y criminología, (16), 1-33. Recuperado de https://bit.ly/35Amrlw

Fernández Molina, E., y Grijalva Eternod, A. (2012). Diseño y validación de dos escalas para medir el miedo al delito y la confianza en la policía. Revista española de investigación criminológica, (10), 1-26. Recuperado de https://bit.ly/34pD3wk

Ferraro, K. y Lagrange, R. (1987). The measurement of fear of crime. Sociological inquiry, 57(1), 70-97. https://doi.org/10.1111/j.1475-682X.1987.tb01181.x.

Fuentealba Carrasco, P., Rojas González, J. y Barriga, O. (2016). Diferencias en la percepción de inseguridad a nivel cognitivo y emocional de acuerdo al perfil sociodemográfico y político: Estudio del Gran Concepción, Chile. Política criminal, 11(22), 620655. http://doi.org/10.4067/S0718-33992016000200008.

Fundación Paz Ciudadana y GFK Adimark (2016). Índice Paz Ciudadana- GfK Adimark. Resultados del Estudio año 2016. Versión conferencia. Recuperado de https://bit.ly/2XVZbvQ

Fundación Paz Ciudadana y GFK Adimark (2017). Índice Paz Ciudadana- GfK Adimark. Resultados del Estudio año 2017. Versión conferencia. Recuperado de https://bit.ly/35MY1pe

Garcia, E., Francisco, J. P. y Caliso, R. A. C. (2017). Reassessing fear of crime: the role of broad insecurities. SSRN electronic journal. https://doi.org/10.2139/ssrn.2895837.

Garofalo, J. (1981). The fear of crime: causes and consequences. The journal of criminal law and criminology, 72(2), 839-857. https://bit.ly/33ujEZM

Gouseti, I. y Jackson, J. (2015). Construal level theory and fear of crime. SSRN electronic journal. https://doi.org/10.2139/ssrn.2545350.

Gray, E., Jackson, J., Farrall, S. (2010). Feelings and functions in the fear of crime: applying a new approach to victimisation insecurity. The British journal of criminology, 51(1). https://doi.org/10.1093/bjc/azq066.

Gray, E., Jackson, J. y Farrall, S. (2011). In search of the fear of crime: using interdisciplinary insights to improve the conceptualisation and measurement of everyday in- 
securities. En D. Gadd, S. Karstedt y S. Messner (Eds.). Sage handbook of criminological research methods (pp. 1-18). Londres: Sage Publications. http://doi.org/10.2139/ssrn.1799251.

Hernández Sampieri, R., Fernández Collado, C. y Baptista Lucio, M. (2014). Metodología de la investigación (6a ed.). México, DF: McGraw-Hill.

Hutton, N. (2005). Beyond populist punitiveness?. Punishment \& society, 7(3), 243-258. https://doi.org/10.1177/1462474505053828.

Jackson, J. y Stafford, M. (2009). Public health and fear of crime: a prospective cohort study. British journal of criminology, 49(6), 832-847. https://doi.org/10.1093/bjc/azp033.

Klama, E. y Egan, V. (2011). The Big-Five, sense of control, mental health and fear of crime as contributory factors to attitudes towards punishment. Personality and individual differences, 51(5), 613-617. https://doi.org/10.1016/j.paid.2011.05.028.

Ley 20.931, de 24 de junio de 2016, Facilita la aplicación efectiva de las penas establecidas para los delitos de robo, hurto y receptación y mejora la persecución penal en dichos delitos, Diario Oficial de la República de Chile, Santiago, 5 de julio de 2016. Recuperado de http://bcn.cl/1wg75

Lim, H. y Chun, Y. (2015). The limitations and advancements in measuring fear of crime. Journal of public administration and governance, 5(2), 140-148. https://doi.org/10.5296/jpag.v5i2.7727.

Lorenc, T., Petticrew, M., Whitehead, M., Neary, D., Clayton, S., Wright, K., ... Renton, A. (2013). Fear of crime and the environment: systematic review of UK qualitative evidence. BMC Public Health, 13(1). https://doi.org/10.1186/1471-2458-13-496.

Marquès i Banqué, M. (2017). Problemas de legitimación del Derecho penal del miedo. Política criminal, 12(24), 690-730. https://doi.org/10.4067/S071833992017000200690.

Medina Ariza, J. (2003). Inseguridad ciudadana, miedo al delito y policía en España. Revista electrónica de ciencia penal y criminología, (5), RECPC 05-03. Recuperado de https://bit.ly/2q1m4Bx

Morales Peillard, A. (2012). La política criminal contemporánea: influencia en Chile del discurso de la ley y el orden. Política criminal, 7 (13), 94-146. https://doi.org/10.4067/S0718-33992012000100003.

Narváez Mora, M. (2015). La identificación del miedo al delito: un límite conceptual en el método de medición. Crítica penal y poder, (8), 94-119. Recuperado de https://bit.ly/2OY0eXQ 
Oviedo S., E. y Rodríguez A., A. (1999). Santiago, una ciudad con temor. Revista panamericana de salud pública, 5(4/5), 278-285. Recuperado de https://bit.ly/34uRPSt

Pain, R. (2000). Place, social relations and the fear of crime: a review. Progress in human geography, 24(3), 365-387. https://doi.org/10.1191/030913200701540474.

Piña Rochefort, J. (2012). Percepción y decisión. Consideraciones teóricas acerca de la relación entre percepción social de inseguridad y conducta delictiva. Revista de estudios de la justicia, (16), 119-130. https://doi.org/10.5354/0718-4735.2013.29501.

Piñera Echeñique, S. (2017). Construyamos tiempos mejores para Chile. Programa de gobierno 2018-2022. Recuperado de https://bit.ly/35IHIPp

Pole, C y Lampard, R. (2002). Practical social investigation: qualitative and quantitative methods in social research. Londres: Prentice Hall.

San Martín, C. (2013). Las representaciones sociales de la seguridad ciudadana en los vecinos de la comuna de Melipilla, Chile.Psicoperspectivas, 12(1), 72-94. https://doi.org/10.5027/psicoperspectivas-Vol12-Issue1-fulltext-219.

Scherman Teilerboim, A. y Etchegaray Thielemann, N. (2013). Consumo de noticias y temor al delito en Chile. Estudios sobre el mensaje periodístico, 19(1), 563-575. https://doi.org/10.5209/rev_ESMP.2013.v19.n1.42539.

Schuermans, N. y De Maesschalck, F. (2010). Fear of crime as a political weapon: explaining the rise of extreme right politics in the flemish countryside. Social \& cultural geography, 11(3), 247-262. https://doi.org/10.1080/14649361003637190.

Skogan, W. (1999). Measuring what matters: Crime, disorder, and fear. En R. Langworthy (Ed.). Measuring what matters: proceedings from the policing research institute meetings (pp. 37-53). Washington, DC: NIJ Cops. Recuperado de https://bit.ly/2DrhXBH

Subsecretaría de prevención del Delito del Ministerio del Interior y Seguridad Pública (2013). Encuesta nacional urbana de seguridad ciudadana. Recuperado de https://bit.ly/2swfDHD

Valera Pertegàs, S. y Guàrdia Olmos, J. (2014). Perceived insecurity and fear of crime in a city with low-crime rates. Journal of environmental psychology, 38, 195-205. https://doi.org/10.1016/j.jenvp.2014.02.002.

Vozmediano Sanz, L., San Juan Guillén, C. y Vergara Iraeta, A. (2008). Problemas de medición del miedo al delito: algunas respuestas teóricas y técnicas. Revista electrónica de ciencia penal y criminología, (10), RECPC 10-07. https://bit.ly/2XUuQO2 
Wainerman, C. y Sautu, R. (Comps.). (2011). La trastienda de la investigación. Buenos Aires: Lumier.

\section{Para citar este artículo bajo Norma APA 6a ed.}

Galleguillos Agurto, S. (2019) Déficits en la medición del miedo al delito en Chile (Coquimbo. En línea), 26, e3860, https://doi.org/10.22199/issn.0718-9753-2019-0015

Copyright del articulo: @2019 Sebastián Galleguillos

(c) BY

Este es un artículo de acceso abierto, bajo licencia Creative Commons BY 4.0. 